\title{
Arbeitsverhältnisse im Dienstleistungssektor
}

\author{
Gerhard Bosch \\ Claudia Weinkopf
}

\begin{abstract}
Der stark expandierende Dienstleistungssektor ist zum Experimentierfeld für prekäre Arbeit geworden. In manchen Dienstleistungsbranchen ist prekäre Arbeit mittlerweile bereits der Normalfall. In diesem Beitrag werden die Verbreitung und die Entwicklungsdynamik schlecht bezahlter und atypischer Arbeitsverhältnisse in unterschiedlichen Dienstleistungsbranchen aufgezeigt. Darüber hinaus wird die Frage diskutiert, mit welchen Maßnahmen man die Qualität von Arbeitsverhältnissen auch in arbeitsintensiven Dienstleistungstätigkeiten verbessern kann.
\end{abstract}

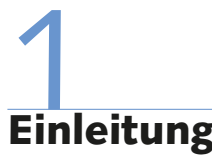

Mittlerweile sind fast drei Viertel (73,5\% im Jahr 2010) aller Beschäftigten in Deutschland im Dienstleistungssektor tätig. Allein in den letzten 20 Jahren (1990-2010) ist der Anteil der Erwerbstätigen im Dienstleistungssektor an allen Erwerbstätigen vor allem auf Kosten der Beschäftigung im sekundären Sektor um 13,6 Prozentpunkte angewachsen. Zugleich hat sich die Zahl der Erwerbstätigen in der Gesamtwirtschaft von 1950 bis 2010 auf knapp 40,5 Mio. mehr als verdoppelt. Hinter dem beeindruckenden Anstieg des Anteils der Dienstleistungsbeschäftigung verbirgt sich ein absoluter Zuwachs von rund 23,4 Mio. Dienstleistungsbeschäftigten innerhalb der letzten 60 Jahre (Statistisches Bundesamt 2011).

Wirtschaftliche Strukturverschiebungen hatten schon immer weitreichende Konsequenzen für die Struktur und Qualität von Arbeitsverhältnissen. Bis in die 1970er Jahre wuchs die Beschäftigung sowohl im sekundären als auch im tertiären Sektor auf Kosten häufig prekärer Beschäftigungsformen. Unsichere und nicht existenzsichernde Beschäftigung war damals im schrumpfenden Bereich der Selbstständigen und mithelfenden Familienangehörigen vor allem in der Landwirtschaft, aber auch den vielen kleinen Läden im Einzelhandel oder ertragsschwachen Handwerks- und Produktionsbetrieben zu finden. Die Beschäftigung wanderte in mittlere und größere Betriebe des sekun- dären und tertiären Sektors ab, die nicht nur eine bessere Bezahlung, sondern auch eine höhere Beschäftigungssicherheit garantieren konnten (Lutz 1984). Durch dieses Aufsaugen prekärer Beschäftigung aus den traditionellen Sektoren wurde das Normalarbeitsverhältnis mit einer unbefristeten Vollzeitbeschäftigung und angemessener, über Tarifverträge ausgehandelten Bezahlung für eine kurze Phase der Vollbeschäftigung auch im Dienstleistungssektor zum Normalfall (Bosch 1986). Da Beschäftigungssicherheit und Bezahlung gesichert waren, konzentrierte sich die Kritik an den Arbeitsverhältnissen in den späten 1960er und 70er Jahren vor allem auf inhumane Arbeitsbedingungen, wie z.B. repetitive und monotone Arbeit sowie hohe körperliche und psychische Belastungen.

Mit der nächsten Phase der Expansion der Dienstleistungsbeschäftigung, die bis heute andauert, veränderte sich die Qualität von Arbeitsverhältnissen grundlegend. Ein Großteil der Beschäftigungsexpansion entfiel auf Teilzeitarbeit und geringfügige Beschäftigung, Leiharbeit, Solo-Selbstständige und befristet Beschäftigte. Seit Mitte der 1990er Jahre expandierte zusätzlich die Niedriglohnbeschäftigung, und im Zuge der Dienstleistungsfreiheit innerhalb der Europäischen Union (EU) wurden zunehmend ausländische Werkvertragsarbeitnehmerinnen und -arbeitnehmer zu den Arbeitsbedingungen ihres Herkunftslandes eingesetzt. Mit Ausnahme von Leiharbeit und Entsendungen, die bis heute ihren Schwerpunkt im Verarbeitenden Gewerbe und in der Bauwirtschaft haben, dienten vor allem Branchen im
Dienstleistungssektor als Experimentierfeld für die Einführung geringer Löhne und die Ausweitung neuer, oft prekärer Beschäftigungsformen.

Die hohe Konzentration schlecht bezahlter Arbeitsverhältnisse im Dienstleistungssektor wirft die Frage auf, ob der Abschied vom Normalarbeitsverhältnis zwar bedauerlich, angesichts des tief greifenden Strukturwandels aber unvermeidlich ist. Solche deterministischen Sichtweisen sind weit verbreitet und auch in manchen Dienstleistungstheorien verankert. Der amerikanische Ökonom Baumol (1967) hat bereits vor mehr als 40 Jahren erstmals auf die „Kostenkrankheit“ (cost desease) von rationalisierungsresistenten Dienstleistungen hingewiesen. Er diagnostizierte vor allem bei arbeitsintensiven personenbezogenen Dienstleistungen solche Rationalisierungsbarrieren. In diesen Branchen könnte daher Beschäftigungswachstum nicht durch rationalisierungsbedingte Preissenkungen wie

Gerhard Bosch, Prof. Dr., ist Geschäftsführender Direktor des Instituts Arbeit und Qualifikation (IAQ) an der Universität DuisburgEssen. Arbeitsschwerpunkte: Arbeitsmarktund Beschäftigungspolitik, Berufliche Bildung, Industrielle Beziehungen, Mindestlöhne.

e-mail: gerhard.bosch@uni-due.de Claudia Weinkopf, Dr., ist Stellvertretende Geschäftsführende Direktorin des IAQ und Leiterin der Forschungsabteilung Flexibilität und Sicherheit. Arbeitsschwerpunkte: Arbeitsmarkt und Beschäftigung, Niedrigund Mindestlöhne, Gender. e-mail: claudia.weinkopf@uni-due.de 
in der Güterproduktion angekurbelt werden. Baumol wollte mit seiner Arbeit auf die drohende Finanzkrise der Kommunen hinweisen, die die Verantwortung für die Finanzierung der meisten personalintensiven Dienstleistungen tragen. Stattdessen wird er heute oft als Kronzeuge für die Schaffung eines Niedriglohnsektors genannt, durch den arbeitsintensive Dienstleistungen verbilligt, zugleich aber auch einkommensschwache Bevölkerungsgruppen von der Inanspruchnahme vieler Dienstleistungen ausgeschlossen werden. Dabei wird übersehen, dass er alternative Strategien, die von einer Finanzierung über öffentliche Mittel oder Sozialversicherungsbeiträge bis hin $\mathrm{zu}$ lohnpolitischen Instrumenten reichen - etwa eine solidarische Lohnpolitik über Branchen hinweg - nie ausgeschlossen hat. Bei Baumol wurde die Kostenkrankheit noch sehr zielgenau in einzelnen Dienstleistungsbranchen verortet. Solche Versuche der Präzisierung sind teilweise zugunsten pauschaler Behauptungen aufgegeben worden. So heißt es z.B. bei Fels et al. (1999) in einem Positionspapier aus dem „Bündnis für Arbeit", das die Agenda 2010 in Deutschland stark beeinflusste, apodiktisch: „Eine Expansion des Dienstleistungssektors kann deshalb wirksam durch eine Senkung der Arbeitskosten gefördert werden“" (ebd. S. 3). Andere Dienstleistungstheorien setzen demgegenüber auf die Schaffung hochwertiger Arbeitsplätze auch in den personalintensiven Dienstleistungen (Bosch/ Lehndorff 2005). Diese Kontroverse ist nicht allein eine akademische, sondern zugleich auch eine höchst praktische Debatte. Letztlich geht es darum, ob die bisher für viele Bereiche industrieller Arbeit geltenden Standards auch künftig in einer Dienstleistungswirtschaft Bestand haben können.

In diesem Beitrag untersuchen wir, wie sich die Arbeitsverhältnisse im Dienstleistungssektor im Vergleich zur Gesamtwirtschaft in den letzten Jahren entwickelt haben. Im Folgenden zeichnen wir zunächst die Entwicklung prekärer Beschäftigung im Dienstleistungssektor nach (Abschnitt 2). In Abschnitt 3 stehen die besonderen Risiken prekärer Arbeit im Vordergrund. Dabei geht es schwerpunkmäßig um Armutsrisiken und die Chancen von Übergängen in nicht prekäre Beschäftigung. Zum Abschluss resümieren wir die Ergebnisse unserer Analyse und zeigen auf, wie man die Qualität von Arbeitsverhältnissen im Dienstleistungssektor verbessern kann (Abschnitt 4).

\section{Prekäre Arbeit im Dienst- leistungssektor}

\subsection{DEFINITION PREKÄRER ARBEIT}

Da Unsicherheit und niedrige Löhne tief ins klassische Normalarbeitsverhältnis eingedrungen sind, reicht die übliche Gegenüberstellung von Normal- und sogenannten atypischen Beschäftigungsverhältnissen längst nicht mehr aus (Weinkopf et al. 2009). Außerdem ist zu berücksichtigen, dass sich unter den „atypischen “ Arbeitsverhältnissen vor allem bei der sozialversicherungspflichtigen Teilzeitarbeit auch gut bezahlte, sozial abgesicherte und auch gewünschte Arbeitsverhältnisse finden. Wenn Teilzeitarbeit zudem mit Optionen auf eine Rückkehr zu Vollzeittätigkeit verbunden ist, kann man sie auch als Vorbote eines neuen Normalarbeitsverhältnisses für Männer und Frauen mit flexiblen Lebensarbeitszeiten und Arbeitszeitoptionen verstehen, wie es in den skandinavischen Ländern zu finden ist. Da im Dienstleistungssektor überwiegend Frauen beschäftigt sind, spiegeln sich in den dortigen Arbeitsverhältnissen auch die Möglichkeiten bzw. Probleme der Vereinbarkeit von Beruf und Familie wider und nicht nur Strategien der Kostenreduzierung von Unternehmen (Bosch 2005).

Wir stützen uns bei der Einordnung prekärer Arbeitsverhältnisse auf eine Definition von Dörre (2005), die nicht nach Erwerbsformen differenziert, sondern die Einhaltung sozialer Standards in den Mittelpunkt stellt: „Ein Arbeitsverhältnis kann als prekär bezeichnet werden, wenn die Beschäftigten aufgrund ihrer Tätigkeit deutlich unter ein Einkommens-, Schutzund soziales Integrationsniveau sinken, das in der Gegenwartsgesellschaft als Standard definiert und mehrheitlich anerkannt wird. Beschäftigungsunsicherheit und Löhne unterhalb des Existenzminimums sind aus der Arbeitskraftperspektive zentrale Merkmale für Prekarität" (ebd. S. 252).

\subsection{VERBREITUNG ATYPISCHER BESCHÄFTIGUNG}

Informationen über die Entwicklung neuer Erwerbsformen und geringer Löhne liefern der jährlich erhobene Mikrozensus und die alle vier Jahre durchgeführte Verdienststrukturerhebung, die allerdings nur Betriebe mit mindestens zehn Beschäftigten umfasst. Über den Mikrozensus sind Langzeitreihen zur Entwicklung der Erwerbsformen im Hauptjob erhältlich. ${ }^{1}$ Als atypische Beschäftigungsverhältnisse werden vom Statistischen Bundesamt Hauptbeschäftigungen in Leiharbeit, befristeten Arbeitsverhältnissen, Minijobs sowie in sozialversicherungspflichtiger Teilzeitarbeit (bis einschließlich 20 Wochenstunden) einbezogen. Die Grundgesamtheit umfasst 15- bis 64-jährige abhängig Beschäftigte, die nicht in Bildung oder Ausbildung sind. Dies ist eine Definition, die das gesamte Ausmaß atypischer Beschäftigung tendenziell unterzeichnet, da auch viele sozialversicherungspflichtige Teilzeitarbeitsverhältnisse keinen ausreichenden Schutz für ein selbstständiges Leben bieten. ${ }^{2}$

Während im Jahr 1996 knapp ein Fünftel aller abhängig Beschäftigten ein atypisches Arbeitsverhältnis hatte, betraf dies im Jahr 2009 bereits fast jeden Vierten. Mit Ausnahme der öffentlichen Verwaltung ist der Anteil atypischer Beschäftigung in allen Wirtschaftsbereichen gestiegen. Der tertiäre Sektor hat mit $25 \%$ (2009) nicht nur einen deutlich höheren Anteil atypischer Beschäftigung als die beiden anderen Sektoren, sondern auch deutlich höhere Zuwächse sowohl in Prozentpunkten als auch in absoluten Zahlen (Tabelle 1). Rund 93 \% des absoluten Wachstums atypischer Beschäftigung seit 1996 entfiel auf Dienstleistungsbranchen. Diese eher statische Sektorenbetrachtung lässt allerdings nicht erkennen, in welchem Ausmaß dieser Zuwachs auf die Auslagerung von und die Nachfrage nach Dienstleistungen aus den beiden anderen Sektoren zurückzuführen ist. So wird zum Beispiel die stark expan-

\footnotetext{
1 Prekäre Nebentätigkeiten, wie sie vor allem bei den Minijobs zu finden sind, werden hier allerdings nicht erfasst. Der Langzeitvergleich enthält für das Jahr 1995 auch nicht die Leiharbeit, die erst in den letzten Jahren stark zugenommen hat.

2 Eine Teilzeitbeschäftigte mit einem Stundenlohn von z.B. $6 €$, die 20 Stunden arbeitet, ist hiernach atypisch beschäftigt, eine Kollegin mit demselben Stundenlohn, die 21 Stunden arbeitet, hat dagegen ein Normalarbeitsverhältnis. Die eine verdient ca. $480 €$ brutto im Monat, die andere $504 €$ Unter Einbeziehung der sozialversicherungspflichtigen Teilzeitarbeit liegt der Anteil atypischer Beschäftigung nach Angaben des Instituts für Arbeitsmarkt- und Berufsforschung bei fast $40 \%$ (IAB 2011).
} 
Tabelle 1: Atypische Arbeitsverhältnisse in den drei Wirtschaftssektoren und den Dienstleistungsbranchen 1996 und 2009 - Zahl und Anteil an den abhängig Beschäftigten -

\begin{tabular}{|c|c|c|c|c|c|c|}
\hline & \multicolumn{2}{|c|}{1996} & \multicolumn{2}{|c|}{2009} & \multicolumn{2}{|c|}{ Veränderung 1996/2009 } \\
\hline & in 1.000 & in $\%$ & in 1.000 & in $\%$ & in 1.000 & (in \%) \\
\hline primärer Sektor & 128 & 13,1 & 122 & 16,2 & -6 & $(-5,7)$ \\
\hline sekundärer Sektor & 1.001 & 8,5 & 1.192 & 11,2 & +191 & $(+19,1)$ \\
\hline $\begin{array}{l}\text { tertiärer Sektor } \\
\text { davon: }\end{array}$ & 3.732 & 18,4 & 5.899 & 25,0 & +2.167 & $(+58,1)$ \\
\hline Handel und Gastgewerbe & 965 & 16,8 & 1.588 & 27,0 & +623 & $(+64,6)$ \\
\hline Verkehr und Nachrichtenübermittlung & 181 & 9,8 & 321 & 16,5 & +140 & $(+77,3)$ \\
\hline Kredit- und Versicherungsgewerbe & 133 & 11,2 & 177 & 14,5 & +44 & $(+33,1)$ \\
\hline $\begin{array}{l}\text { Grundstückswesen, Vermietung, } \\
\text { wirtschaftliche Dienstleistung }\end{array}$ & 397 & 18,9 & 893 & 23,5 & +540 & $(+224,9)$ \\
\hline Öffentliche Verwaltung u.ä. & 470 & 16,6 & 400 & 16,0 & -70 & $(+14,9)$ \\
\hline $\begin{array}{l}\text { Öffentliche und persönliche } \\
\text { Dienstleistungen }\end{array}$ & 1.586 & 24,2 & 2.520 & 30,4 & +934 & $(+58,9)$ \\
\hline Gesamtwirtschaft & 4.861 & 19,7 & 7.213 & 23,4 & +2.370 & $(+48,4)$ \\
\hline
\end{tabular}

dierende Leiharbeit, die schwerpunktmäßig im verarbeitenden Gewerbe genutzt wird, in Statistiken unter „Wirtschaftliche Dienstleistungen“ gebucht.

Auch zwischen den Wirtschaftsbereichen im Dienstleistungssektor sind erhebliche Unterschiede erkennbar. In der öffentlichen Verwaltung und im Kredit- und Versicherungsgewerbe liegt der Anteil atypischer Beschäftigung nur wenig höher als im sekundären Sektor. Die größte Bedeutung hat atypische Beschäftigung im Bereich öffentlicher und persönlicher Dienstleistungen (gut $30 \%$ ), im Handel und Gastgewerbe (27 \%) sowie im Bereich wirtschaftlicher Dienstleistungen $(23,5 \%)$. In diesen drei Wirtschaftsbereichen konzentrieren sich mit 5 Mio. Personen fast $70 \%$ aller atypisch Beschäftigten.

\subsection{NIEDRIGLÖHNE NACH BRANCHEN}

Insbesondere in den expandierenden Dienstleistungsbranchen ist auch der Niedriglohnanteil von einem ohnehin schon hohen Niveau seit 1995 noch einmal stark angewachsen. In Tabelle 2 sind auf Basis der Verdienststrukturerhebung von 2006 die Anteile von Niedriglohnbeschäftigten nach Branche, Geschlecht und Erwerbsform aufgeschlüsselt. Tabelle 2 zeigt zunächst, dass niedrige Löhne für Männer in einem Normalarbeitsverhältnis nur eine vergleichsweise geringe Rolle spielen, während bei Frauen das Niedriglohnrisiko mehr als doppelt so hoch liegt. Es ist weiterhin zu erkennen, dass fast jeder zweite atypisch Beschäftigte bzw. jede zweite nur einen Niedriglohn erhält gegenüber etwa jedem neunten Beschäftigten in einem Normalarbeitsverhältnis. Auch innerhalb der atypischen Erwerbsformen ist das Niedriglohnrisiko unterschiedlich hoch. Während mehr als vier Fünftel aller Minijobberinnen und Minijobber und mehr als zwei Drittel aller Leiharbeitskräfte gering entlohnt werden, ist bei den befristet Beschäftigten gut jeder Dritte von einem Niedriglohn betroffen. Der Niedriglohnan-

Tabelle 2: Anteil der Beschäftigten*) mit Niedriglohn - nach Geschlecht und Wirtschaftszweig im Oktober 2006 in \% -

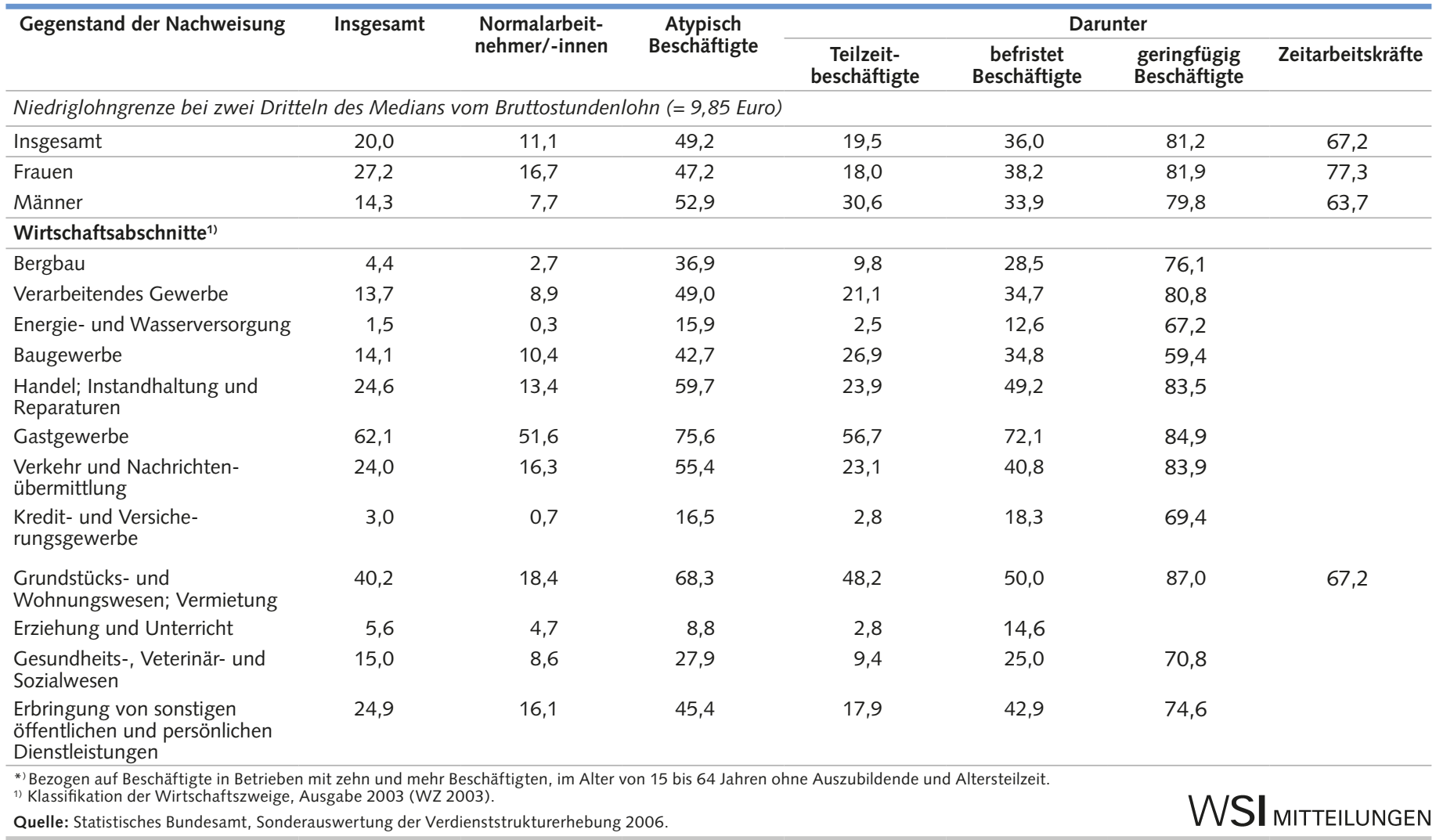


teil unter den sozialversicherungspflichtig Teilzeitbeschäftigten liegt mit knapp $20 \%$ zwar deutlich niedriger, aber immer noch fast doppelt so hoch wie bei den in einem Normalarbeitsverhältnis Beschäftigten.

Ein genauerer Blick auf die Branchenverteilung zeigt die hohe Spannbreite in der Entlohnung der Erwerbsformen. Im Kredit- und Versicherungsgewerbe, im Bereich Erziehung und Unterricht sowie im Gesundheits-, Veterinär- und Sozialwesen haben Beschäftigte in sozialversicherungspflichtiger Teilzeitarbeit mit Niedriglohnanteilen unterhalb des Niveaus von Normalarbeitnehmern in der Gesamtwirtschaft ein vergleichsweise geringes Niedriglohnrisiko. Teilzeitarbeit wird hier meist nach Tarif entlohnt, ist oft auch freiwillig gewählt und zum Teil - wie etwa im öffentlichen Dienst - mit Rückkehrrechten auf Vollzeit verbunden. In diesen Dienstleistungsbranchen sind auch befristet Beschäftigte deutlich seltener als im Durchschnitt der Gesamtwirtschaft von Niedriglöhnen betroffen, was mit der hohen Tarifbindung und den überdurchschnittlichen Löhnen in diesen Bereichen zusammenhängen dürfte.

Das Bild wandelt sich jedoch völlig, wenn man auf die anderen Branchen schaut. Im Gastgewerbe erhält auch die Mehrheit der Normalbeschäftigten einen Niedriglohn. Die klassische Definition des Normalarbeitsverhältnisses greift hier also nicht mehr. Auch in anderen Dienstleistungsbranchen wie dem Einzelhandel, dem Grundstücks- und Wohnungswesen sowie der Verkehrs- und Nachrichtenübermittlung liegt das Niedriglohnrisiko von Normalarbeitnehmerinnen und -nehmern deutlich über dem Durchschnitt aller Branchen.

Internationale Vergleiche zeigen, dass in Deutschland nicht nur die Niedriglohnbeschäftigung stärker als in den meisten anderen europäischen Ländern angestiegen ist. Durch das Fehlen von wirkungsvollen Lohnuntergrenzen ist das Lohnspektrum in Deutschland auch stark nach unten ausgefranst. Im Jahr 2008 verdienten fast 5 Mio. Beschäftigte (15,7\%) weniger als $8 €$ die Stunde, was in unseren westlichen Nachbarländern angesichts der dortigen Mindestlöhne nicht legal wäre (Kalina/Weinkopf 2010). Hinzu kommt, dass inzwischen fast $80 \%$ der Niedriglohnbeschäftigten in Deutschland eine abgeschlossene Berufsausbildung oder einen akademischen Abschluss haben, während in anderen Ländern überwiegend gering Qualifizierte von Niedriglöhnen betroffen sind. ${ }^{3}$ Wenn selbst eine formale Qualifikation oft nicht vor geringer Bezahlung schützt, sinken die Anreize von Beschäftigten, eine Berufsausbildung zu absolvieren oder sich beruflich fortzubilden, und es drohen Qualitätsverluste in den Niedriglohnbranchen. Vor allem im Gastgewerbe, der Branche mit dem höchsten Niedriglohnanteil, bröckelt die Bereitschaft Jugendlicher, eine Lehre zu beginnen oder die Beschäftigung nach $\mathrm{Ab}$ schluss der Ausbildung in dieser Branche fortzusetzen (Vanselow 2007).

Für den raschen Anstieg niedrig entlohnter Beschäftigung in der Gesamtwirtschaft und insbesondere im Dienstleistungssektor gibt es eine Reihe von Ursachen: Die Erhöhung der Erwerbsquote von Frauen in den letzten Jahrzehnten hat im konservativen westdeutschen Wohlfahrtsstaat als eine Art eingebauter Deregulator des traditionellen gut bezahlten Normalarbeitsverhältnisses gewirkt. Frauenarbeit wurde in diesem Modell lange nur als Zusatztätigkeit verstanden, die folglich auch geringer bezahlt werden kann. Im Unterschied zu den skandinavischen Ländern mit ihrer solidarischen Lohnpolitik, die über die Wirtschaftszweige hinweg Gültigkeit hat, war es niemals Ziel der deutschen Gewerkschaften, die Lohnunterschiede zwischen den klassischen Männer- und Frauenbranchen zu verringern. Hinzu kommen die immer noch großen Schwierigkeiten, Beruf und Familie in Deutschland miteinander zu vereinbaren. Die wachsende Anzahl erwerbstätiger Frauen mit Kindern kann oft nur einen Minijob oder eine kurze sozialversicherungspflichtige Teilzeitarbeit im Dienstleistungssektor ausüben, aus denen die Frauen dann nicht oder nur unter großen Schwierigkeiten herauskommen.

Weitere Triebkräfte waren und sind die Privatisierung ehemals öffentlicher Dienstleistungen, das Outsourcing von Tätigkeiten aus tarifgebundenen Unternehmen in tariffreie Zonen oder in Dienstleistungsbranchen mit niedrigeren Löhnen, das Fehlen von wirkungsvollen Lohnuntergrenzen und die abnehmende Bedeutung allgemeinverbindlicher Tarifverträge. Eine wichtige Rolle spielen außerdem die Deregulierung von Beschäftigungsverhältnissen (vor allem bei der Leiharbeit und den Minijobs) sowie veränderte Unternehmensstrategien mit einer stärkeren
Nutzung atypischer Erwerbsformen zur Absenkung von Löhnen einschließlich grenzüberschreitender Werkaufträge. Hinzu kommt die durch Steuersenkungen und Beitragsbegrenzungen herbeigeführte Unterfinanzierung des Staates und der Sozialversicherungen, die eine angemessene Entlohnung vor allem bei den arbeitsintensiven sozialen Dienstleistungen erschwert (Bosch/Wagner 2002; Lehndorff 2006).

Die Vielfalt dieser Ursachen deutet schon an, dass es längst nicht allein um eine Schlechterstellung atypischer Beschäftigungsformen an den Rändern des Beschäftigungssystems geht, sondern zugleich auch um die Arbeitsbedingungen im ehemals gut bezahlten Kern der Beschäftigten in Normalarbeitsverhältnissen.

\section{Risiken prekärer Dienst- leistungsarbeit}

\subsection{AUFSTOCKUNG GERINGER EINKOMMEN}

Prekäre Dienstleistungsbeschäftigung ist mit hohen Armutsrisiken verbunden. Im März 2010 waren fast 1,4 Mio. Arbeitslosengeld (ALG)-II-Beziehende zwar erwerbstätig, aber ihr Arbeitseinkommen reichte nicht aus, um damit den haushaltsbezogenen Mindestbedarf zu decken. Dies kann zwar auch an kurzen Arbeitszeiten oder einem hohen Bedarf - etwa durch mehrere Kinder - liegen, aber geringe Stundenlöhne spielen hierbei eine wichtige Rolle. In solchen Fällen besteht ein Rechtsanspruch, dass das zu geringfügige Einkommen aus Erwerbsarbeit auf das Niveau der Grundsicherung für Arbeitsuchende aufgestockt wird. Eine Studie des IAB zeigt, dass fast $30 \%$ der westdeutschen und $40 \%$ der ostdeutschen „Aufstockerinnen und Aufstocker" weniger als $5 €$ brutto pro Stunde verdienen (Dietz et al. 2009).

In den Statistiken der Bundesagentur für Arbeit wird bezogen auf diese Gruppe nur zwischen sozialversicherungspflich-

\footnotetext{
3 Voss-Dahm (2009) hat eindrucksvoll dargestellt, dass viele qualifizierte Frauen in der Familienphase den subventionierten Minijobs im Einzelhandel noch die "Treue halten“. Mit der Veränderung des Familienmodells in Deutschland wird dieses billige Arbeitskräftereservoir perspektivisch jedoch schrumpfen.
} 
tiger und geringfügiger Beschäftigung unterschieden. Ein Vergleich der Sektoren zeigt zunächst, dass die Anteile der Aufstockerinnen und Aufstocker im tertiären Sektor mit 3,2 \% bei den sozialversicherungspflichtig und $15,6 \%$ bei den geringfügig Beschäftigten deutlich über den entsprechenden Werten im sekundären Sektor liegen (Tabelle 3). Besonders hohe Anteile von Beschäftigten mit ergänzenden ALG-II-Ansprüchen innerhalb des Dienstleistungssektors sind im Gastgewerbe und in der Arbeitnehmerüberlassung erkennbar, also (wie erwartet) in Branchen mit besonders hohen Niedriglohnanteilen. Insgesamt reicht bei fast 1,2 Mio. Beschäftigten im Dienstleistungssektor das Erwerbseinkommen nicht aus, um den Lebensunterhalt zu bestreiten, und mehr als die Hälfte von ihnen sind sozialversicherungspflichtig beschäftigt. Die ebenfalls überdurchschnittlichen Werte der Aufstocker mit einem Minijob im Handel, Gastgewerbe und in der Arbeitnehmerüberlassung weisen zusätzlich auf hohe Anteile von Arbeitsverträgen mit geringem Stundenvolumen und somit eine ausgeprägte Fragmentierung von Beschäftigungsverhältnissen hin.

\section{2 ÜBERGÄNGE AUS PREKÄRER ARBEIT}

Häufig wird argumentiert, dass viele atypisch Beschäftigte anderweitig abgesichert

\section{Tabelle 3: Erwerbstätige ALG-II-Beziehende (Aufstocker) nach Sektor und Dienstleistungsbereichen}

- Zahl und Anteil an den Beschäftigten, März 2010 -

\begin{tabular}{|c|c|c|c|c|c|c|}
\hline \multirow[b]{3}{*}{ Primärer Sektor } & \multicolumn{3}{|c|}{$\begin{array}{c}\text { Sozialversicherungspflichtig } \\
\text { Beschäftigte }\end{array}$} & \multicolumn{3}{|c|}{ Minijob } \\
\hline & gesamt & $\begin{array}{c}\text { davon mit } \\
\text { ALG II- } \\
\text { Anspruch }\end{array}$ & in $\%$ & gesamt & $\begin{array}{c}\text { davon mit } \\
\text { ALG II- } \\
\text { Anspruch }\end{array}$ & in $\%$ \\
\hline & 200.596 & 7.033 & $3,5 \%$ & 59.659 & 6.446 & $10,8 \%$ \\
\hline Sekundärer Sektor & 8.240 .424 & 86.318 & $1,0 \%$ & 515.891 & 60.498 & $11,7 \%$ \\
\hline Tertiärer Sektor & 18.703 .702 & 603.334 & $3,2 \%$ & 3.736 .248 & 583.225 & $15,6 \%$ \\
\hline \multicolumn{7}{|l|}{ Darunter } \\
\hline Handel & 3.972 .698 & 104.643 & $2,6 \%$ & 905.659 & 114.064 & $12,6 \%$ \\
\hline Verkehr & 1.361 .142 & 43.235 & $3,2 \%$ & 255.070 & 54.546 & $21,4 \%$ \\
\hline Gastgewerbe & 796.436 & 68.945 & $8,7 \%$ & 494.117 & 116.696 & $23,6 \%$ \\
\hline luk & 822.902 & 8.269 & $1,0 \%$ & 127.103 & 16.063 & $12,6 \%$ \\
\hline Finanzen & 999.978 & 3.233 & $0,3 \%$ & 42.574 & 2.500 & $5,9 \%$ \\
\hline Arbeitnehmerüberlassung & 591.792 & 67.905 & $11,5 \%$ & 49.918 & 10.721 & $21,5 \%$ \\
\hline $\begin{array}{l}\text { Wirtschaftliche DL ohne } \\
\text { ANÜ }\end{array}$ & 2.839 .410 & 118.585 & $4,2 \%$ & 792.958 & 147.960 & $18,7 \%$ \\
\hline Öffentliche Verwaltung & 1.699 .888 & 13.884 & $0,8 \%$ & 60.779 & 4.590 & $7,6 \%$ \\
\hline Erziehung & 1.094 .595 & 33.701 & $3,1 \%$ & 143.865 & 7.518 & $5,2 \%$ \\
\hline Gesundheit & 3.449 .349 & 85.073 & $2,5 \%$ & 414.599 & 40.531 & $9,8 \%$ \\
\hline Sonstige DL & 1.075 .512 & 55.861 & $5,2 \%$ & 449.606 & 68.036 & $15,1 \%$ \\
\hline
\end{tabular}

Sozialpolitisch gesehen sind kurzzeitige Phasen von prekärer Beschäftigung im Erwerbsverlauf weniger problematisch, als wenn sich Niedriglöhne auf bestimmte Personengruppen konzentrieren, die nur geringe Chancen haben, in besser bezahlte Tätigkeiten aufzusteigen. In Deutschland wird der Ausbau des Niedriglohnsektors häufig damit gerechtfertigt, dass gering bezahlte Jobs einen Einstieg in den Arbeitsmarkt ermöglichen und sich die Beschäftigten im Laufe der Zeit in besser bezahlte Beschäftigungsverhältnisse hocharbeiten können. Anfang der 1990er Jahre war die Einkommensmobilität - also der Aufstieg in eine besser bezahlte Tätigkeit - in Deutschland tatsächlich größer als etwa in den USA oder Großbritannien (OECD 1997, S. 31; Keese et al. 1998, S. 249). Mit der Expansion atypischer Arbeit und der Zunahme der Niedriglohnbeschäftigung hat sich dies jedoch geändert. Die Chancen, dass gering bezahlte Jobs als Sprungbrett in bessere Beschäftigung fungieren, haben sich in den letzten Jahren in Deutschland deutlich verschlechtert (Bosch/Kalina 2007; Kalina 2008; Schank et al. 2008), während sie in anderen Ländern mit geringen Niedriglohnanteilen wie etwa Dänemark weiterhin hoch sind (Mason/ Salverda 2010).

Ähnlich enttäuschend sind die Ergebnisse hinsichtlich der Übergänge aus atypischer Beschäftigung. Gensicke et al. (2010) zeigen, dass die Übergangsquoten aus geringfügiger Beschäftigung, Teilzeitarbeit, befristeter Beschäftigung und Leiharbeit in ein Normalarbeitsverhältnis gering sind. Zu vergleichbaren Ergebnissen kommen auch detailliertere Untersuchungen $\mathrm{zu}$ einzelnen Formen atypischer Arbeit. So gelangen z.B. Crimmann et al. (2009) zu dem Schluss, „dass Leiharbeit weniger als Brücke in reguläre Beschäftigung dient, sondern eher der Start für eine dauerhafte „Zeitarbeitskarriere“ ist. Zwei Drittel der Personen, die 2004 ein Leiharbeitsverhältnis eingingen, schaffen in den beiden darauffolgenden Jahren nicht den Sprung in nachhaltige Beschäftigung, sondern fassen lediglich Fuß in der Arbeitnehmerüberlassung. Mit 80 Prozent verbleibt sogar der Großteil derjenigen, die bereits einmal in der Arbeitnehmerüberlassung tätig waren, in dieser Branche" (ebd. S. 131).

Niedriglohnjobs und atypische Beschäftigung erfüllen offenbar immer weniger die Funktion, den Weg in reguläre und gut bezahlte Beschäftigung zu ebnen. 
Langfristig verringern sich damit auch die Chancen der betroffenen Beschäftigten, im Alter über eine auskömmliche Rente zu verfügen. Die abnehmende Aufwärtsmobilität ist zum einen Folge veränderter Unternehmensstrategien. Durch die Auslagerung schlecht bezahlter Tätigkeiten in andere Unternehmen, wie etwa bei der Reinigung, dem Catering oder den Wachund Sicherheitsdiensten, wird der Zugang zu besser bezahlten Tätigkeiten in den internen Arbeitsmärkten der beauftragenden Unternehmen verbaut (vgl. z.B. Vanselow 2007). Hinzu kommen starke institutionelle Barrieren gegen einen Einkommensaufstieg im deutschen Beschäftigungssystem. Da Leiharbeitskräfte nicht den gleichen Lohn wie Stammkräfte erhalten müssen, haben die entleihenden Unternehmen nur geringe Anreize, sie fest anzustellen. Bei Minijobs gibt es weder für die Beschäftigten noch für die Unternehmen Anreize zur Überschreitung der Geringfügigkeitsgrenze. Die Beschäftigten verlieren ihre abgeleitete Krankenversicherung sowie Vorteile aus dem Ehegattensplitting. Die Unternehmen, die Minijobberinnen und Minijobber in der Praxis trotz des geltenden Gleichbehandlungsgebotes oft geringer entlohnen und ihnen weder bezahlten Urlaub noch Lohnfortzahlung im Krankheitsfall gewähren, wollen ihre Lohnkosten nicht erhöhen. Auch selbst gewählte Teilzeitarbeit kann zur Falle werden. Vollzeitbeschäftigte haben zwar das Recht, ihre Arbeitszeit zu verringern und Teilzeit zu arbeiten, doch umgekehrt gibt es kein Anrecht auf den Wechsel von Teilzeit- auf Vollzeitarbeit.

Die Wirkungen atypischer und prekärer Beschäftigung beschränken sich nicht allein auf die unmittelbar Betroffenen, sondern lassen auch den Kern der Beschäftigten nicht unberührt (Dörre 2005, S. 254). Die Verfügbarkeit kostengünstiger Beschäftigungsverhältnisse tangiert auch die Arbeits- und Entlohnungsbedingungen der Stammbeschäftigten. Eine zunehmende Nutzung z.B. von Leiharbeit oder Minijobs oder das Outsourcing von Tätigkeiten kann oft nur um den Preis erheblicher Zugeständnisse verhindert werden (Rehder 2003). Eine Neujustierung der Kosten und Risiken atypischer bzw. prekärer Beschäftigung liegt daher nicht allein im Interesse der unmittelbar Betroffenen, sondern aller Beschäftigten.

Ein Blick auf andere Länder zeigt, dass die Prekarität von Dienstleistungstätigkeiten in Deutschland besonders ausgeprägt ist. Der Unterschied zwischen den Arbeitskosten im Dienstleistungssektor und im Verarbeitenden Gewerbe ist nur in wenigen anderen EU-Ländern so groß wie in Deutschland. So lagen die Arbeitskosten pro Stunde im Verarbeitenden Gewerbe im Jahr 2009 mit 33,10 € im Durchschnitt $6,60 €$ über den Arbeitskosten im privaten Dienstleistungssektor. Die Differenz betrug in Frankreich nur 0,60€, in Italien $0,70 €$, in Großbritannien $1,10 €$ und in Schweden 1,20€ (Niechoj et al. 2011). Ähnliche Unterschiede zeigen internationale Vergleiche zur Niedriglohnbeschäftigung (Mason/Salverda 2010) oder zu den Lohnunterschieden zwischen Männern und Frauen (Hausmann et al. 2009).

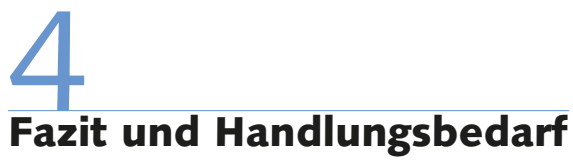

Atypische und gering bezahlte Beschäftigungsverhältnisse haben in Deutschland an Bedeutung gewonnen und spielen im Dienstleistungssektor eine erheblich größere Rolle als im sekundären Sektor. In einigen Dienstleistungsbereichen wie etwa dem Gastgewerbe betreffen prekäre Arbeitsformen sogar eine deutliche Mehrheit der Beschäftigten und sind dort zum Normalfall geworden. Das Ausmaß der Prekarität variiert je nach Beschäftigungsform und weiteren Arbeitsplatzmerkmalen und wird auch dadurch beeinflusst, wie gut oder schlecht die Chancen auf Übergänge in andere Beschäftigungsformen sind.

Um die Qualität von Arbeitsverhältnissen im Dienstleistungssektor zu erhöhen, ist aus unserer Sicht ein Bündel von Maßnahmen erforderlich, das nicht alleine am Arbeitsmarkt ansetzen kann. Ein erster Ansatzpunkt ist die Finanzierung von personenbezogenen Dienstleistungen, durch die sich schon von der Angebotsseite her das Ausmaß prekärer Beschäftigung beeinflussen lässt. Würden solche Dienstleistungen allein über den Markt abgewickelt, könnten sie von vielen eigentlich Bedürftigen nicht in Anspruch genommen werden. Dies hätte bei Bildungs-, Gesundheits- und Pflegedienstleistungen jedoch erhebliche negative Auswirkungen auf die Qualität des Humankapitals, den Wohlstand sowie auf den sozialen Zusammenhalt der Gesellschaft. Durch Sozialversicherungen wie im Bereich der Pflege und Gesundheit, durch einkommensabhängige Beiträge wie teil- weise in der Kinderbetreuung oder durch eine kostenfreie öffentliche Bereitstellung wie in der schulischen Bildung lässt sich jedoch die „Kostenkrankheit“ dieser Dienste neutralisieren. Durch solche Instrumente kann nicht nur das Beschäftigungswachstum gefördert, sondern auch der Zugang aller Einkommensgruppen zu diesen für Lebensqualität, Humankapitalbildung und Wachstum wichtigen Dienstleistungen sichergestellt werden.

Ein zweiter Ansatzpunkt ist die Verankerung von Mindeststandards im Beschäftigungssystem. Durch einen gesetzlichen Mindestlohn würden wirkungsvolle Lohnuntergrenzen gesetzt, die vor allem in den Niedriglohnbranchen des Dienstleistungssektors zu Lohnanhebungen im unteren Bereich führen würden. Die neuere US-amerikanische und britische empirische Mindestlohnforschung hat gezeigt, dass dadurch keine Beschäftigung verloren geht (Bosch et al. 2009). Mindestlöhne alleine garantieren aber noch keine angemessene Bezahlung für qualifizierte Arbeit. Frankreich, Belgien und die Niederlande haben daher gesetzliche Mindestlöhne mit allgemeinverbindlichen Tarifverträgen kombiniert. In den skandinavischen Ländern entfalten die Tarifverträge infolge der hohen Gewerkschaftsdichte vergleichbare Wirkungen. Auch in Deutschland waren die Tarifverträge z.B. im Einzelhandel bis 2000 allgemeinverbindlich. Diese Tradition muss wiederbelebt werden, weil die Gefahr besteht, dass das berufliche Bildungssystem in wichtigen Dienstleistungsbranchen zusammenbricht, wenn man auch mit einer beruflichen Ausbildung nur einen Niedriglohn erzielen kann.

Ein dritter Ansatzpunkt bezieht sich auf eine bessere Regulierung atypischer Beschäftigungsverhältnisse. Eine wesentliche Triebkraft der Zunahme atypischer Beschäftigung in Deutschland besteht darin, dass es in der Praxis oft hohe Lohnabschläge im Vergleich zu regulären Beschäftigten, die ähnliche Tätigkeiten ausführen, gibt. Bei sozialversicherungspflichtiger Teilzeitarbeit, Minijobs und befristeten Arbeitsverhältnissen steht dies im Widerspruch zu den Vorgaben im Teilzeit- und Befristungsgesetz, die eine Lohndiskriminierung explizit untersagen. Bei Leiharbeitskräften findet sich der Equal Pay-Grundsatz zwar im Arbeitnehmerüberlassungsgesetz, wird aber durch die Klausel, dass tarifvertragliche 
Abweichungen zulässig sind, ausgehöhlt. Bei Leiharbeit sollte daher wie in anderen europäischen Ländern der Grundsatz der gleichen Bezahlung wie bei den Beschäftigten des Entleihbetriebs gelten. Weiterhin ist eine Abschaffung der Sonderregelungen für Minijobs zwingend notwendig, wie sie auch vom Deutschen Juristentag (Waltermann 2010), vom Sachverständigenrat (2009), vom Deutschen Frauenrat (2010) und im Gleichstellungsbericht der Bundesregierung (2011) gefordert wird. Ziel muss sein, alle Arbeitsverhältnisse in die Sozialversicherungen einzubeziehen. Durch die Gleichbehandlung atypischer Erwerbsformen würden nicht nur die An- reize zur Umwandlung regulärer in atypische Beschäftigung verringert, sondern auch die Chancen für Übergänge in ein Normalarbeitsverhältnis steigen.

Weiterer Handlungsbedarf besteht bei der Ausgestaltung und Absicherung vor allem von Teilzeitarbeit und hinsichtlich der Verankerung eines neuen Leitbildes für die Erwerbstätigkeit von Männern und Frauen im Lebensverlauf. Wenn Männer und Frauen gleichermaßen erwerbstätig sind, müssen die Erwerbsverläufe flexibilisiert werden, um Beruf und Familie miteinander vereinbaren zu können. Notwendig ist ein neues Normalarbeitsverhältnis, das auf Vollzeitarbeit basiert, aber Optionen zur Unterbrechung oder Verringerung der Erwerbstätigkeit in Phasen der Erziehung, Pflege oder Weiterbildung bietet. Selbst gewählte Teilzeitarbeit und Sabbaticals wären dann durchaus normale, nicht diskriminierte Phasen in Erwerbsverläufen. Im Gleichstellungsbericht der Bundesregierung (2011, S. 222) wird vorgeschlagen, solche Optionen in einem „Gesetz über Wahlarbeitszeiten“ zu bündeln. Ein solches modernisiertes Normalarbeitsverhältnis erfordert nicht nur Rückkehroptionen aus Teilzeit und Erwerbsunterbrechungen, sondern durch den Ausbau der Kinderbetreuung und von Ganztagsschulen auch eine tatsächliche Wahlfreiheit.

\section{LITERATUR}

Baumol, W.J. (1967): Macroeconomics of Unbalanced Growth: The Anatomy of Urban Crisis, in: American Economic Review 57 (3), S. $416-426$

Bosch, G. (1986): Hat das Normalarbeitsverhältnis eine Zukunft?, in: WSI-Mitteilungen 39 (3), S. 163-176

Bosch, G. (2005): Das deutsche Beschäftigungsmodell zwischen Dekommodifizierung und Vermarktlichung, in: Kurz-Scherf, I./Correll, L./ Janczyk, S. (Hrsg.): In Arbeit: Zukunft. Die Zukunft der Arbeit und der Arbeitsforschung liegt in ihrem Wandel, Münster, S. 86-102

Bosch, G./Kalina, T. (2007): Niedriglohnbeschäftigung in Deutschland, in: Bosch, G./Weinkopf, C. (Hrsg.): Arbeiten für wenig Geld: Niedriglohnbeschäftigung in Deutschland, Frankfurt a. M., S. 20-105

Bosch, G./Lehndorff, S. (2005): Introduction: service economies - high road or low road?, in: Bosch, G./Lehndorff, S. (Hrsg.): Working in the service sector: a tale from different worlds, London, S. 1-31

Bosch, G./Wagner, A. (2002): Nachhaltige Dienstleistungspolitik, in: Bosch, G./Hennicke, P./Hilbert, J./Kristof, K./Scherhorn, G. (Hrsg.): Die Zukunft von Dienstleistungen: ihre Auswirkung auf Arbeit, Umwelt und Lebensqualität, Frankfurt a. M., S. 482-512

Bosch, G./Weinkopf, C./Kalina, T. (2009): Mindestlöhne in Deutschland, Wiso Diskurs - Expertisen und Dokumentationen zur Wirtschafts- und Sozialpolitik, hrsg. von der Friedrich-Ebert-Stiftung, Bonn

Bundesagentur für Arbeit (2011): Arbeitsmarkt in Zahlen. Erwerbstätige Arbeitslosengeld-II-Bezieher, Juni 2010, Nürnberg

Crimmann, A./Ziegler, K./Ellguth, P./Kohaut, S./Lehmer, F. (2009): Forschungsbericht zum Thema "Arbeitnehmerüberlassung", Bundesministerium für Arbeit und Soziales, Forschungsbericht Arbeitsmarkt 397, Berlin
Deutscher Frauenrat (2010): Minijobs - Wege in die Armut. Kann sich unsere Gesellschaft Minijobs leisten? Positionspapier des Deutschen Frauenrates zu Minijobs, beschlossen auf der Mitgliederversammlung 2010, Berlin

Dietz, M./Müller, G./Trappmann, M. (2009): Bedarfsgemeinschaften im SGB II. Warum Aufstocker trotz Arbeit bedürftig bleiben, IAB-Kurzbericht (2), Nürnberg

Dörre, K. (2005): Prekarität - eine arbeitspolitische Herausforderung, in: WSI-Mitteilungen 58 (5), S. 250-258

Fels, G./Heinze, R./Pfarr, H./Streeck, W. (1999): Bericht der Wissenschaftlergruppe der Arbeitsgruppe Benchmarking über Möglichkeiten zur Verbesserung der Beschäftigungschancen gering qualifizierter Arbeitnehmer, Berlin

Gensicke, M./Herzog-Stein, A./Seifert, H./Tschersich, N. (2010): Einmal atypisch, immer atypisch beschäftigt? Mobilitätsprozesse atypischer und normaler Arbeitsverhältnisse im Vergleich, in: WSI-Mitteilungen 63 (4), S. 179-187, www.boeckler.de/119_103164.html Gleichstellungsbericht der Bundesregierung (2011): Neue Wege Gleiche Chancen. Gleichstellung von Frauen und Männern im Lebensverlauf, Gutachten der Sachverständigenkommission an das Bundesministerium für Familie, Senioren, Frauen und Jugend für den ersten Gleichstellungsbericht der Bundesregierung, München

Hausmann, R./Tyson, L. D./Zahidi S. (2009): The global gender report, World Economic Forum, Genf

Institut für Arbeitsmarkt- und Berufsforschung (IAB) (2011): „Das Normalarbeitsverhältnis ist kein Auslaufmodell “, Presseinformation des Instituts für Arbeitsmarkt- und Berufsforschung vom 3.3., Nürnberg Kalina, T. (2008): Niedriglohnbeschäftigung in Deutschland: Sprungbrett oder Sackgasse?, in: Arbeit 17 (1), S. 21-37 
Kalina, T./Weinkopf, C. (2008): Konzentriert sich die steigende Niedriglohnbeschäftigung in Deutschland auf atypisch Beschäftigte?, in: Zeitschrift für Arbeitsmarktforschung 41 (4), S. 447-469

Kalina, T./Weinkopf, C. (2010): Niedriglohnbeschäftigung 2008 : Stagnation auf hohem Niveau - Lohnspektrum franst nach unten aus, IAQ-Report (06), Duisburg

Keese, M./Puymoyen, A./Swain, P. (1998): The incidence and dynamics of low-paid employment in OECD countries, in: Asplund, R./ Sloane, P. J./Theodossiou, I. (Hrsg.): Low pay and earnings mobility in Europe, Cheltenham/Northampton, S. 223-265

Lehndorff, S. (2006): Motor der Entwicklung - oder fünftes Rad am Wagen? Soziale Dienstleistungen als gesellschaftliche Investitionen, in: Lehndorff, S. (Hrsg.): Das Politische in der Arbeitspolitik: Ansatzpunkte für eine nachhaltige Arbeits- und Arbeitszeitgestaltung, Berlin, S. 249-277

Lutz, B. (1984): Der kurze Traum immerwährender Prosperität: Eine Neuinterpretation der industriell-kapitalistischen Entwicklung im Europa des 20. Jahrhunderts, Frankfurt/New York

Mason, G./Salverda, W. (2010): Low pay: Living standards and employment, in: Gautié, J./Schmitt, J. (Hrsg.): Low-wage work in the wealthy world, New York, S. 35-90

Niechoj, T./Stein, U./Stephan, S./Zwiener, R. (2011): Deutsche Arbeitskosten und Lohnstückkosten im europäischen Vergleich Auswirkungen der Krise, IMK Report (60), Düsseldorf OECD (1997): Employment Outlook, Paris

Rehder, B. (2003): Betriebliche Bündnisse für Arbeit in Deutschland. Mitbestimmung und Flächentarif im Wandel, Frankfurt

Schank, T./Schnabel, C./Stephani, J. (2008): Geringverdiener: Wem und wie gelingt der Aufstieg?, IAB-Discussion Paper (14), Nürnberg
Sachverständigenrat zur Begutachtung der gesamtwirtschaftlichen Entwicklung (2009): Atypische Beschäftigungsverhältnisse: Zweiklassengesellschaft am Arbeitsmarkt? (Ziffern 513 bis 530), Auszug aus dem Jahresgutachten 2008/2009, Wiesbaden, S. 301-311 Statistisches Bundesamt (2011): Erwerbstätige im Inland nach Wirtschaftssektoren, Deutschland, www.destatis.de/jetspeed/portal/ cms/Sites/destatis/Internet/DE/Content/Statistiken/Zeitreihen/ LangeReihen/Arbeitsmarkt/Content75//rerw13a,templateld= renderPrint.psml

Vanselow, A. (2007): Immer noch verloren und vergessen - Zimmerreinigungskräfte in Hotels, in: Bosch, G./Weinkopf, C. (Hrsg.): Arbeiten für wenig Geld: Niedriglohnbeschäftigung in Deutschland, Frankfurt, S. $211-248$

Voss-Dahm, D. (2009): Über die Stabilität sozialer Ungleichheit im Betrieb: Verkaufsarbeit im Einzelhandel, Berlin

Waltermann, R. (2010): Abschied vom Normalarbeitsverhältnis? Welche arbeits- und sozialrechtlichen Regelungen empfehlen sich im Hinblick auf die Zunahme neuer Beschäftigungsformen und die wachsende Diskontinuität von Erwerbsbiographien? Gutachten für den 68. Deutschen Juristentag, Bonn

Weinkopf, C./Hieming, B./Mesaros, L. (2009): Prekäre Beschäftigung. Expertise für die SPD-Landtagsfraktion NRW, Institut Arbeit und Qualifikation, Duisburg

Wingerter, C. (2009): Der Wandel der Erwerbsformen und seine Bedeutung für die Einkommenssituation Erwerbstätiger, in: Wirtschaft und Statistik (11), S. 1080-1098 